\title{
DLX4 as a prognostic marker for hepatocellular carcinoma
}

\author{
Y. GAO ${ }^{1}$, Z. $\mathrm{LI}^{1}$, X. GUO 2 , Y. LIU' 1 K. ZHANG 3 ,* \\ ${ }^{1}$ Department of Diagnostic Ultrasound, 302 Hospital of PLA; ${ }^{2}$ Pathological Diagnosis and Research Canter; 302 Hospital of PLA; ${ }^{3}$ Department \\ of Medical Affairs, 302 Hospital of PLA, Beijing, P.R. China \\ ${ }^{*}$ Correspondence: kaibozhang@yeah.net
}

Received August 23, 2013 / Accepted September 18, 2013

\begin{abstract}
Distal-less homeobox gene 4 (DLX4) has been shown to play a role in carcinogenesis. The value of DLX4 in hepatocellular carcinoma (HCC) has not been fully investigated. Here, we sought to quantify the expression of DLX4 in association with the clinicopathological features and prognosis in hepatocellular carcinoma (HCC). We examined DLX4 expression in 45 HCC tissues and matched adjacent noncancerous tissues by quantitative RT-PCR (qRT-PCR) and Western blotting. Furthermore, 226 HCC samples were analyzed for DLX4 expression by immunohistochemistry. The expression of DLX4 was significantly elevated in HCC tissues in comparison with that in adjacent non-cancerous tissues $(\mathrm{P}<0.01)$. The high expression of DLX4 in HCC was significantly correlated with tumor size, histopathologic classifications, and serum alpha-fetoprotein (AFP). Kaplan-Meier survival analysis revealed that increased expression of DLX4 correlated with poor clinical outcomes of HCC patients $(\mathrm{P}<0.01)$. Subsequent multivariate analysis showed that DLX4 expression was an independent prognostic biomarker for overall survival of HCC patients. These results suggest that high expression of DLX4 predicts HCC prognosis, and DLX4 may be potentially an important therapeutic target for HCC.
\end{abstract}

Key words: DLX4, hepatocellular carcinoma, prognosis, marker

The frequency of hepatocellular carcinoma (HCC) ranks fifth among all malignancies worldwide and is the third leading cause of cancer-related death [1]. Hepatectomy is the first choice to offer long-term survival benefit for HCC patients. However, high recurrence and mortality rate still remains a major problem for HCC [2]. Thus, exploring predictive factors for HCC prognosis will help to screen high-risk factors affecting patient prognosis and develop better treatments for HCC management.

Homeobox genes encode transcription factors that regulate cell differentiation [3]. The Distal-less (DLX) subfamily of homeobox genes includes six identified members referred to as DLX 1-6 in the mouse and DLX 1-6 in humans [4]. Substantial experimental data support the notion that the absence of expression of members of the Dlx/DLX family results in embryonic lethality in mouse and altered expression of these genes may play a role in human tumorigenesis $[5,6]$. Expression patterns of members of the Dlx genes have been well characterized in the developing embryo [7] and especially in organ substructures that are dependent on epithelial mesenchymal cell interactions for their formation [8]. Distal-less homeobox gene 4 (DLX4) is a member of the DLX4 family of homeobox genes that controls many aspects of embryonic development [9]. Although most normal adult tissues are negative for DLX4, multiple tumors including leukemias, lung, breast, ovarian and prostate cancers show positive expression of DLX4 [10]. Recently, DLX4 has been reported to be associated with epithelial to mesenchymal transition (EMT) and tumor metastasis [11]. However, it remains to be investigated whether DLX4 plays a role in HCC. Considering DLX4 is expressed in diverse types of tumors, we investigated the possible involvement of DLX4 in HCC. Our study demonstrated that protein expression of DLX4 in HCC tissues was significantly higher as compared to that in adjacent non-cancerous tissues. The high expression of DLX4 in cancer was significantly correlated with clinical outcomes.

\section{Patients and methods}

Patients and tissue samples. To investigate DLX4 expression in HCC, 45 HCC cancerous tissues and paired adjacent non-cancerous tissues ( $\geq 2 \mathrm{~cm}$ away from HCC) were collected from HCC cancer patients undergoing hepatectomy at our hospital between January and June, 2009. The histological 
diagnosis of HCC was confirmed by two independent pathologists. Surgically removed fresh tissues were immediately frozen in liquid nitrogen and stored at $-80^{\circ} \mathrm{C}$. In addition, the other group included 226 consecutive paraffin-embedded HCC tissues which were collected between 2003 and 2007. All these patients had undergone hepatic resection in our Department.

The follow-up data of all patients enrolled in this study were obtained according to routine medical records. We performed postoperative follow-up on HCC patients every 3 months during the first 2 years, every 6 months during the 3 rd to the 5 th year, and thereafter annually for additional 5 years or until death. Overall survival (OS) was defined as the time from surgery to patient's death or the last follow up. Disease free survival (DFS) was defined as the time from surgery to disease recurrence or metastasis. The histological types of HCC were defined according to the classification criteria of WHO. This study was approved by the Institutional Ethics Board of our hospital, and written informed consent was obtained from all participants.

Quantitative RT-PCR (qRT-PCR). Total RNA was extracted using TRIzol reagent (Qiagen, China) according to manufacturer's protocol. An equal amout of RNA $(8 \mu \mathrm{g})$ was reversely transcribed into cDNA by Reverse Transcriptase (Invitrogen, USA) according to the manufacturer's instructions.

DLX4 and GAPDH were then amplified by quantitative real-time PCR using the following primers: DLX4: forward: 5'-CCCTCTGGGATCTACCCAAGG-3', reverse: 5'-TGATGCTGATACCAGGCTCCA-3'; GAPDH: forward: 5'-CTCCTCCTGTTC GACAGTCAGC-3', reverse: 5'-CCCAATACGACCAAATCCGTT-3'. Gene-specific amplification was performed in an ABI 7900HT real-time PCR system using SYBR Green master mix (Invitrogen, USA). The mixture was preheated for $10 \mathrm{~min}$ at $95^{\circ} \mathrm{C}$ and followed by 40 cycles of amplification $\left(30 \mathrm{~s}\right.$ at $95^{\circ} \mathrm{C}$ and $1 \mathrm{~min}$ at $58^{\circ} \mathrm{C}$, respectively). The CT value of each sample was calculated, and the relative expression of DLX4 mRNA was normalized to the GAPDH (2- $\triangle \mathrm{Ct}$ method).

Western blot analysis. The frozen HCC or non-cancerous tissue samples were homogenated in a RIPA buffer (Qiagen, China). After centrifugation at $12,000 \mathrm{rpm}, 4^{\circ} \mathrm{C}$ for $20 \mathrm{~min}$, $60 \mu \mathrm{g}$ of protein samples was run on a $12 \%$ SDS-PAGE gel and transferred to polyvinylidene difluoride membrane (Millipore). After blocking non-specific binding sites for $60 \mathrm{~min}$ with $5 \%$ non-fat milk, the membranes were incubated with rabbit monoclonal antibody against DLX4 (1:1,000; LifeSpan BioSciences, Inc.), and GAPDH (1:1,000;Santa Cruz) at $4^{\circ} \mathrm{C}$ over night, respectively. Then, membranes were washed with TBST for three times, 15 min each time and then incubated with HRP-conjugated anti-rabbit secondary antibody (1:10,000; Santa Cruz) for $60 \mathrm{~min}$ at room temperature. The membrane was developed by an enhanced chemiluminescence system (ECL; Millipore) after washed with TBST for three times. The intensity of the protein bands was determined by densitometry using Image J software.
Immunohistochemistry. Paraffinic-embedded tissues from 226 HCC patients were subjected for immunohistochemistry analysis. The slides were immersed in EDTA $(\mathrm{pH} \mathrm{8.0)}$ and boiled for $20 \mathrm{~min}$ in the microwave oven for the antigen retrieval. After rinse with PBS, endogenous peroxidase was blocked with $0.3 \%$ hydrogen peroxide for $20 \mathrm{~min}$ at room temperature. The slides were incubated with the anti-DLX4 (1:50; LifeSpan BioSciences, Inc.) in a humidified chamber at $4^{\circ} \mathrm{C}$ overnight. Following additional wash with PBS for three times, the sections were sequentially incubated with horseradish peroxidase-conjugated secondary antibody (LifeSpan BioSciences, Inc.) at $37^{\circ} \mathrm{C}$ for $30 \mathrm{~min}$ and then washed three times with PBS. Finally, diaminobenzidine tetrahydrochloride (DAB) was used for the signal development. PBS was used as negative control.

The total DLX4 immunostaining scores were calculated as the sum of percent positivity of stained tumor cells and the staining intensity. The percent of positivity was scored as "0" $(<5 \%$, negative), "1" (5-25\%), "2" (25-50\%), "3" (50$75 \%), " 4$ " (>75\%). The staining intensity was scored as "0" (no staining), "1" (weak staining), "2"(moderate staining), and " 3 " (strong staining). Both percent positivity of cells and staining intensity were determined under double-blind conditions. The DLX4 immunostaining score was calculated with the value of percent positivity score plus staining intensity score, ranged from 0 to 12 . DLX 4 expression level was defined as following: '-'(score 0-3), ' + ' (score 4-6), '++' (score 7-9), and' +++ ' (score $\geq 10)$. Based on the DLX4 expression levels, the HCC patients were divided into two groups: low DLX4 expression $(-$ and +$)$ and high DLX4 expression (++ and +++).

Statistical analysis. Statistical analyses were performed using SPSS16.0. Student t-test was used to compare protein expression of DLX4 in HCC. Correlations of DLX4 expression with clinicopathological features were evaluated by Chi-square test. Follow-up time was censored if the follow-up was lost. Survival curves were calculated using the Kaplan-Meier method and compared by the log-rank test. Univariate and multivariate analyses were used to explore the association of DLX4 expression with HCC clinicopathological variables and patient's survival. $\mathrm{P}<0.05$ were considered significant.

\section{Results}

mRNA expression of DLX4 in HCC tissues. To test the DLX4 mRNA expression level, we used qRT-PCR to detect 45 fresh surgical HCC cancerous samples and the paired adjacent non-cancerous tissues from the same patients. As a result, the DLX4 mRNA expression level was significantly higher in cancerous tissues than that in corresponding adjacent noncancerous tissues (Fig. 1, $\mathrm{P}<0.001$ ). All the 45 -paired tissues were tested in triplicate.

Protein expression of DLX4 in HCC tissues. Further, to support the results of the qRT-PCR analysis, we explore the expression of DLX4 at the protein level using Western blotting on the same 45-paired HCC cancerous tissues and their cor- 


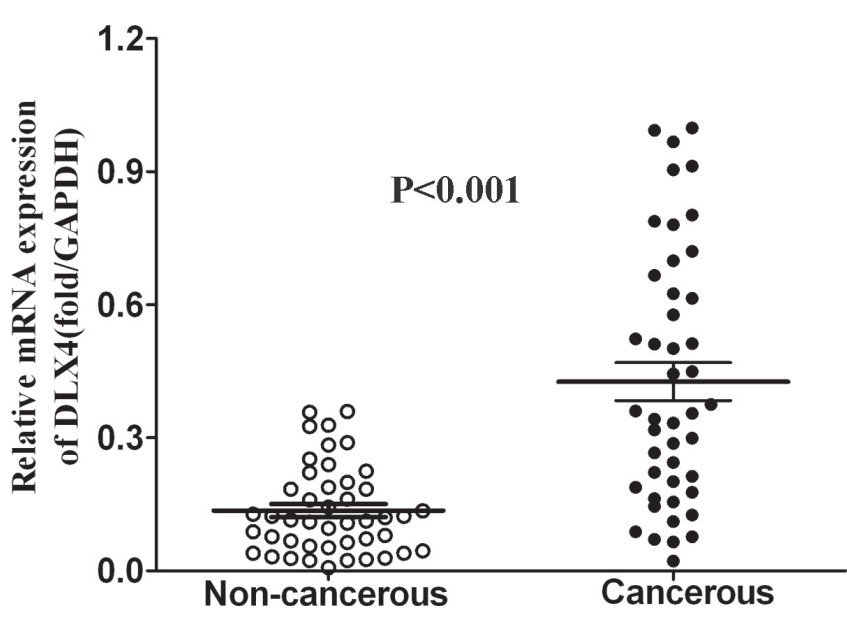

Figure 1. Up-regulated mRNA expression of DLX4 in 45 hepatocellular carcinoma tissues compared to matched adjacent non-cancerous tissues as assessed by quantitative RT-PCR.

Table 1. Correlations of DLX4 expression with clinicopathologic features of hepatocellular carcinoma

\begin{tabular}{|c|c|c|c|c|}
\hline \multirow{2}{*}{$\begin{array}{l}\text { Clinicopathologic } \\
\text { variables }\end{array}$} & \multirow{2}{*}{$\begin{array}{l}\text { Number } \\
\text { of cases }\end{array}$} & \multicolumn{2}{|c|}{ DLX4 expression } & \multirow{2}{*}{ P-Value } \\
\hline & & Low & High & \\
\hline \multicolumn{5}{|l|}{ Gender } \\
\hline Male & 191 & 85 & 106 & \\
\hline Female & 35 & 11 & 24 & 0.49 \\
\hline \multicolumn{5}{|l|}{ Age (years) } \\
\hline$\leq 60$ & 185 & 78 & 107 & \\
\hline$>60$ & 41 & 18 & 23 & 0.53 \\
\hline \multicolumn{5}{|l|}{ Liver cirrhosis } \\
\hline Yes & 167 & 59 & 108 & \\
\hline No & 59 & 37 & 22 & 0.45 \\
\hline \multicolumn{5}{|l|}{ Serum AFP } \\
\hline$\geq 400 \mu \mathrm{g} / \mathrm{l}$ & 121 & 37 & 68 & \\
\hline$<400 \mu \mathrm{g} / 1$ & 105 & 59 & 62 & 0.015 \\
\hline \multicolumn{5}{|l|}{ Tumor size } \\
\hline$\leq 5 \mathrm{~cm}$ & 94 & 61 & 71 & \\
\hline$>5 \mathrm{~cm}$ & 132 & 35 & 59 & 0.034 \\
\hline \multicolumn{5}{|c|}{ Histological differentiation } \\
\hline Well & 63 & 26 & 37 & \\
\hline Moderate & 66 & 29 & 37 & 0.023 \\
\hline Poor & 97 & 41 & 56 & \\
\hline \multicolumn{5}{|l|}{ Recurrence } \\
\hline Presence & 161 & 72 & 89 & \\
\hline Absence & 65 & 24 & 41 & 0.34 \\
\hline \multicolumn{5}{|l|}{ Metastasis } \\
\hline Presence & 157 & 59 & 98 & \\
\hline Absence & 69 & 37 & 32 & 0.012 \\
\hline
\end{tabular}

Note: Clinicopathologic variables were compared between group with negative DLX4 expression ( $\mathrm{n}=96)$ and group with positive DLX4 expression $(n=130)$.
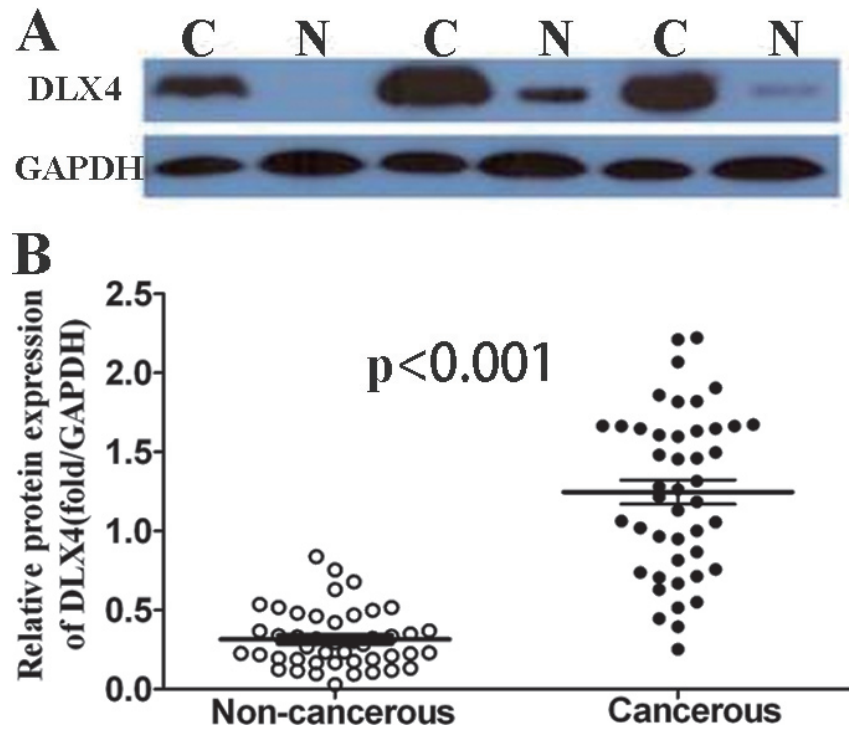

Figure 2 Increased protein expression of DLX4 in hepatocelluar cancer tissues compared to matched adjacent non-cancerous tissues as determined by Western blotting. A. Representative images of DLX4 protein expression in hepatocelluar cancer tissues and the matched adjacent non-cancerous tissues from 3 patients. B. All of the hepatocelluar cancer tissues and the matched adjacent noncancerous tissues from 45 patients.

responding adjacent non-cancerous tissues. Consistent with the mRNA expression level, Western blot analyses showed that DLX4 expression was markedly elevated in HCC cancerous tissues in comparison with their corresponding non-cancerous tissues $(\mathrm{P}<0.001$, Fig. 2$)$.

Relationship of DLX4 expression with clinicopathological variables in HCC. To investigate the correlations between DLX4 expression and clinicopathological variables in HCC, immunohistochemical staining were performed in 226 HCC specimens. Among them, $57.9 \%$ of HCC cancerous samples (130/226) showed high expression of DLX4 (Fig. 3). However, DLX4 expression was found negative or low in all 153 non-paired adjacent liver tissues. The relationships between DLX4 expression and clinicopathological features are presented in Table 1. As shown, the DLX4 expression was significantly correlated with serum AFP $(\mathrm{P}=0.015)$, tumor size $(\mathrm{P}=0.034)$, histological differentiation $(\mathrm{P}=0.023)$ and metastasis $(\mathrm{P}=0.012)$. We found no statistical differences between DLX4 expression and age, gender, liver cirrhosis, or recurrence $(\mathrm{P}>0.05)$.

Relationship between DLX4 expression and patients' survival. Furthermore, we evaluated the prognostic value of DLX4 expression on OS and DFS of HCC patients between patients with high and low expression of DLX4. Kaplan-Meier curve analysis indicated that high DLX4 expression was significantly correlated with poor prognosis of HCC patients. HCC patients with high DLX4 expression had obviously shorter OS and DFS as compared with those with low DLX4 expression (Fig. 4). 

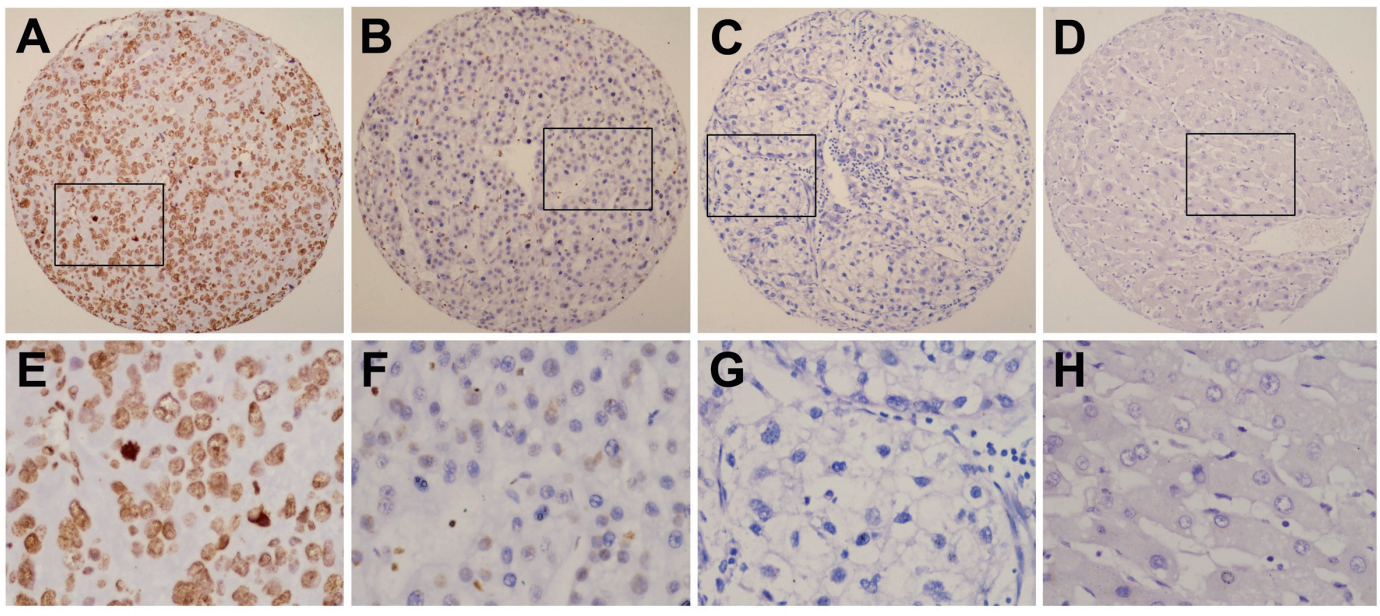

Figure 3. The DLX4 expression in hepatocelluar cancer and adjacent non-cancerous tissues by immunohistochemistry. A.High DLX4 expression (100x). B. Low DLX4 expression (100×). C.Negative DLX4 expression in an HCC case (100×). D. Negative DLX4 expression in adjacent non-cancerous liver tissues $(100 \times)$. (E), (F), (G) and (H) demonstrate the $400 \times$ from the area from (A), (B), (C) and (D), respectively.

Univariate and multivariate analyses of prognostic variables in HCC patients. Cox proportional-hazard model (univariate and multivariate analyses) was performed to investigate the impacts of DLX4 expression pattern on clinicopathological features of HCC patients. Univariate analysis showed that DLX4 expression, histological grading and tumor size were significant prognostic factors (Table 2). Based on multivariate Cox regression analysis, DLX4 was an independent prognostic factor. Thus, DLX4 expression may play a role in predicting the overall survival in HCC patients $(\mathrm{P}=0.005$, Table 2).

\section{Discussion}

In the present study, we demonstrated the evidence that elevated expression of DLX4 protein is correlated with poor prognosis of HCC patients. Our data demonstrate that DLX4 is up-regulated at both mRNA and protein levels in HCC tissues as compared with adjacent normal liver tissues. Paired HCC lesions and adjacent noncancerous tissues displayed significantly different expression levels of DLX4, with the cancer lesions displaying obviously higher expression of DLX4. Consistently, immunohistochemical analysis also showed that DLX4 expression was increased in most HCC cancerous tissues compared with their paired non-cancerous tissues. Immunohistochemistry analysis indicated that the high DLX4 expression was strongly correlated with aggressiveness of HCC including tumor size, histological differentiation and metastasis as well as serum AFP level in patients with HCC. Our data implicate that high DLX4 expression may be a common feature in HCC and participates in the progression of HCC. Investigating the role of DLX4 expression in HCC may aid in the development of new diagnostic and therapeutic strategies.

Table 2. Univariate and multivariate analysis of overall survival in hepatocellular carcinoma patients

\begin{tabular}{lcccccc}
\hline Variables & \multicolumn{3}{c}{ Univariate analysi } & \multicolumn{3}{c}{ Multivariate analysis } \\
\cline { 2 - 7 } & HR & $95 \%$ CI & $P$ & HR & $95 \%$ CI & $P$ \\
\hline DLX4 & 3.07 & $1.09-6.45$ & $<0.001$ & 2.04 & $1.04-3.89$ & 0.005 \\
Gender & 0.65 & $0.28-1.52$ & 0.33 & & & \\
Age & 0.81 & $0.48-1.37$ & 0.44 & & & \\
Tumor size & 1.49 & $1.13-2.43$ & 0.019 & 1.21 & $0.88-1.93$ & 0.085 \\
Histology & 1.60 & $1.06-2.56$ & 0.011 & 1.34 & $1.05-2.17$ & 0.066 \\
Cirrhosis & 0.78 & $0.52-1.11$ & 0.22 & & & \\
HBsAg & 1.32 & $0.42-2.27$ & 0.74 & & & \\
Serum AFP & 1.592 & $0.92-2.74$ & 0.09 & & & \\
Metastasis & 1.01 & $0.69-2.23$ & 0.28 & & & \\
Recurrence & 1.25 & $0.70-2.09$ & 0.31 & & & \\
\hline
\end{tabular}

HR: Hazard ratio; CI: Confidence interval; AFP: $\alpha$-fetoprotein. 

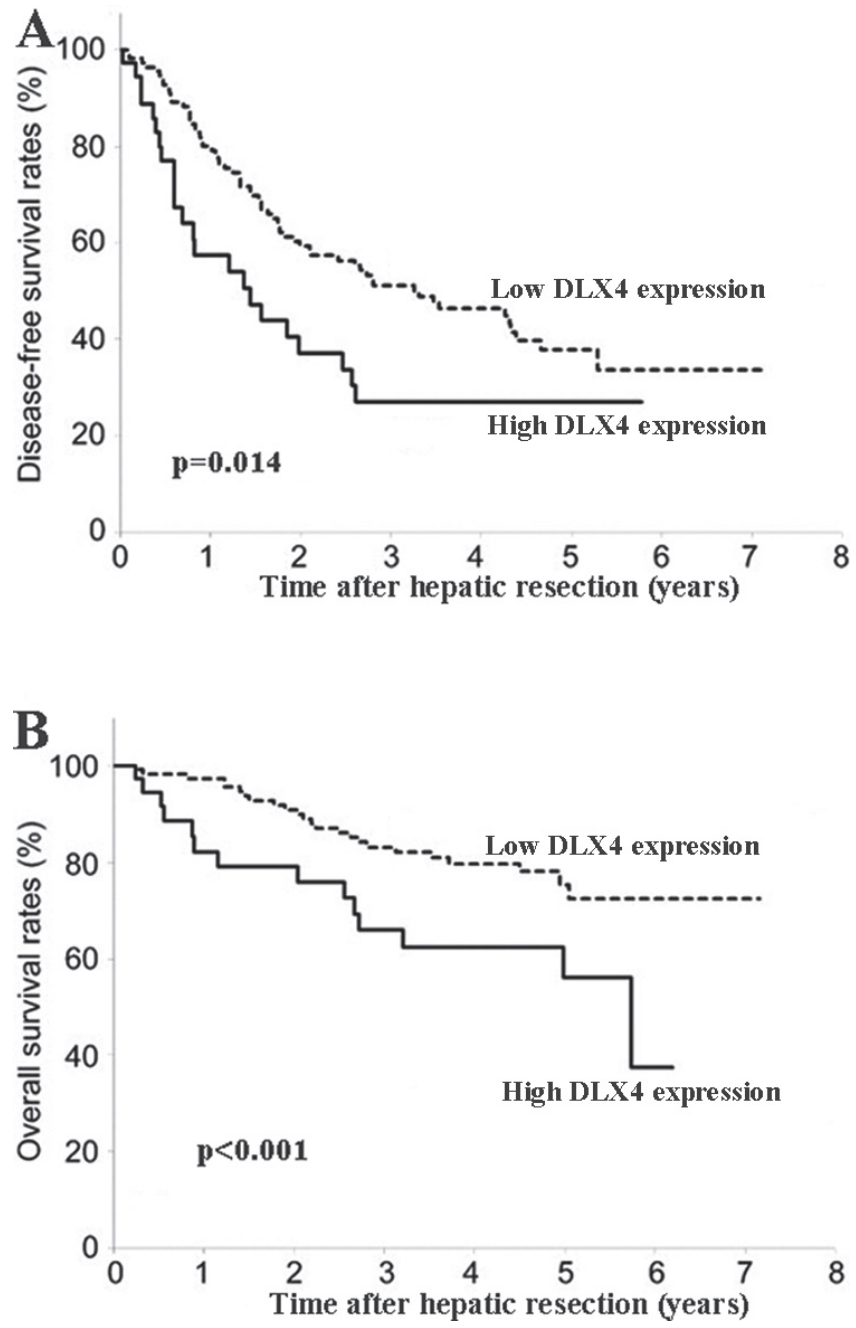

Figure 4. High DLX4 expression was correlated with poor prognosis of hepatocellular carcinoma patients. A. Kaplan-Meier survival analysis of disease-free survival in 226 HCC patients between the high DLX4 expression and the low DLX4 expression by log-rank test. B, Kaplan-Meier survival analysis of overall survival in $226 \mathrm{HCC}$ patients between the high DLX4 expression and the low DLX4 expression by log-rank test.

Our data also demonstrated that the high DLX4 expression was associated with unfavorable prognosis. Of note, DLX4 is an independent prognostic indicator in HCC. DLX4 expression was correlated with both OS and DFS of HCC patients. In agreement with the present results, DLX4 was upregulated in multiple cancers [10]. The percentage of nuclei expressing both DLX4 is increased in breast carcinoma [12].The prevalence of DLX 4 positive cells and the intensity of DLX 4 immunoreactivity increased with the extent of ductal proliferation and carcinogenesis and DLX4 positive cells had a substantially higher proliferation rate than morphologically similar cells without DLX4 expression, suggesting that expression of DLX4 may reliably reflect or directly contribute to tumor progression and/or invasion [13]. A study by Sun et al. suggests that DLX4 may be involved in the survival of human choriocarcinoma cells, which may be mediated by the inhibition of apoptosis [14].Yu et al reported that DLX4 might contribute to breast neoplastic transformation or tumor progression and suggest that DLX4 mRNA level has potential as a prognostic predictor for breast cancer [15]. Clinically, DLX4 levels were elevated in all pretreatment acute promyelocytic leukemia patients tested, while DLX4 expression was decreased in $91 \%$ of patients after combined chemotherapy treatment, what suggests that DLX4 may be part of a pathway involved in resistance to therapy and that DLX4 is a potential therapeutic target in acute promyelocytic leukemia [16]. DLX4 mRNA was expressed at significantly higher levels in non-small cell lung cancer tissues than in adjacent non-cancerous tissues and normal lung samples and patients with high DLX4 mRNA expression had a poorer prognosis in terms of both DFS and OS [17]. Additionally, DLX4 mRNA expression level was an independent prognostic factor for DFS. Schwartz et al. suggest that that DLX4 is an important upstream factor in the carcinogenic pathway of prostate cancer and that the expression of DLX4 may reflect or directly contribute to tumor progression and/or invasion [18].

In conclusion, this study demonstrates for the first time that high DLX4 expression is up-regulated in the majority of HCC tissues at both transcriptional and translational levels. And our data establish DLX4 as a novel unfavorably prognostic biomarker of HCC patients.

\section{References}

[1] Jemal A, Bray F, Center MM, Ferlay J, Ward E, et al. Global cancer statistics. CA Cancer J Clin 2011; 61: 69-90. http: //dx.doi.org/10.3322/caac.20107

[2] Bruix J, Sherman M. Management of hepatocellular carcinoma: an update. Hepatology 2010; 53: 1020-2. http://dx.doi. org/10.1002/hep.24199

[3] McGinnis W, Krumlauf R. Homeobox genes and axial patterning. Cell. 1992; 68: 283- 302. http://dx.doi.org/10.1016/ 0092-8674(92)90471-N

[4] Simeone A, Acampora D, Pannese M, D`Esposito M, Stornaiuolo A, et al. Cloning and characterization of two members of the vertebrate Dlx gene family. Proc Natl Acad Sci USA 1994; 91: 2250-4. http: //dx.doi.org/10.1073/ pnas.91.6.2250

[5] Ferrari N, Palmisano GL, Paleari L, Basso G, Mangioni M, et al. DLX genes as targets of ALL-1: DLX 2,3,4 down-regulation in $\mathrm{t}(4 ; 11)$ acute lymphoblastic leukemias. J Leukoc Biol 2003; 74: 302-5. http: //dx.doi.org/10.1189/jlb.1102581

[6] Morasso MI, Radoja N. Dlx genes, p63, and ectodermal dysplasias. Birth Defects Res C Embryo Today 2005; 75: 163-71. http: //dx.doi.org/10.1002/bdrc.20047

[7] Quint E, Zerucha T, Ekker M. Differential expression of orthologous Dlx genes in zebrafish and mice: implications for the evolution of the Dlx homeobox gene family. J Exp Zool 2000; 288: 235-41. http: //dx.doi.org/10.1002/1097010X(20001015)288: $3<235:$ : AID-JEZ4>3.0.CO; 2-J 
[8] Murthi P, Said JM, Doherty VL, Donath S, Nowell CJ, et al. Homeobox gene DLX4 expression is increased in idiopathic human fetal growth restriction. Mol Hum Reprod. 2006; 12: 763-9. http: //dx.doi.org/10.1093/molehr/gal087

[9] Panganiban G, Rubenstein JL. Developmental functions of the Distal-less/DLX4 homeobox genes. Development 2002; 129: 4371-86.

[10] Trinh BQ, Barengo N, Naora H. Homeodomain protein DLX4 counteracts key transcriptional control mechanisms of the TGF- $\beta$ cytostatic program and blocks the antiproliferative effect of TGF- $\beta$. Oncogene. 2011; 30: 2718-29. http: //dx.doi. org/10.1038/onc.2011.4

[11] Zhang L, Yang M, Gan L, He T, Xiao X, et al. DLX4 upregulates TWIST and enhances tumor migration, invasion and metastasis. Int J Biol Sci 2012; 8: 1178-226. http: //dx.doi. org/10.7150/ijbs.4458

[12] Neufing PJ, Kalionis B, Horsfall DJ, Ricciardelli C, Stahl J, et al. Expression and localization of homeodomain proteinsDLX4/ HB9 in normal and malignant human breast tissues. Anticancer Res 2003; 23: 1479-88.

[13] Man YG, Fu SW, Schwartz A, Pinzone JJ, Simmens SJ, et al. Expression of BP1, a novel homeobox gene, correlates with breast cancer progression and invasion. Breast Cancer Res
Treat 2005; 90: 241-7. http://dx.doi.org/10.1007/s10549-004$\underline{4492-9}$

[14] Sun Y, Lu X, Yin L, Zhao F, Feng Y. Inhibition of DLX4 promotes apoptosis in choriocarcinoma cell lines. Placenta. 2006; 27: 375-83. http: //dx.doi.org/10.1016/j.placenta.2005.03.007

[15] Yu M, Yang Y, Shi Y, Wang D, Wei X, et al. Expression level of beta protein $1 \mathrm{mRNA}$ in Chinese breast cancer patients: a potential molecular marker for poor prognosis. Cancer Sci 2008; 99: 173-8.

[16] Awwad RT, Do K, Stevenson H, Fu SW, Lo-Coco F, et al. Overexpression of BP1, a homeobox gene, is associated with resistance to all-trans retinoic acid in acute promyelocytic leukemia cells. Ann Hematol. 2008; 87: 195-203. http: //dx.doi. org/10.1007/s00277-007-0402-7

[17] Yu M, Wan Y, Zou Q. Prognostic significance of BP1 mRNA expression level in patients with non-small cell lung cancer. Clin Biochem 2008; 41: 824-30. http: //dx.doi.org/10.1016/ j.clinbiochem.2008.03.011

[18] Schwartz AM, Man YG, Rezaei MK, Simmens SJ, Berg PE. $\mathrm{BP} 1$, a homeoprotein, is significantly expressed in prostate adenocarcinoma and is concordant with prostatic intraepithelial neoplasia. Mod Pathol. 2009; 22: 1-6. http: //dx.doi. org/10.1038/modpathol.2008.168 